\title{
miR-302d-3p regulates the viability, migration and apoptosis of breast cancer cells through regulating the TMBIM6-mediated ERK signaling pathway
}

\author{
YANRU LIAO ${ }^{1}$, ZHENXIONG QIU ${ }^{2}$ and LING BAI $^{3}$ \\ Departments of ${ }^{1}$ Thyroid and Breast Surgery, ${ }^{2}$ General Surgery and ${ }^{3}$ Pathology and Central Laboratory, \\ Baoan Central Hospital of Shenzhen, Shenzhen, Guangdong 518102, P.R. China
}

Received June 22, 2020; Accepted February 5, 2021

DOI: $10.3892 / \mathrm{mmr} .2021 .12493$

\begin{abstract}
MicroRNAs (miRs/miRNAs) play important roles in the occurrence, metastasis and prognosis of multiple types of cancers. However, the specific role of miR-302d-3p and its underlying mechanism in breast cancer $(\mathrm{BC})$ have not yet been reported. The present study aimed to identify the role of miR-302D-3p in BC and its potential mechanism using BC cell lines MCF7 and MDA-MB-231 and normal breast epithelial cell MCF-10A. Cancer and paracancerous tissue from patients with $\mathrm{BC}$ were also used. Reverse transcription-quantitative PCR was performed to detect the expression of miR-302d-3p and transmembrane Bax inhibitor motif containing 6 (TMBIM6). Dual-luciferase reporter assays verified the binding sites of miR-302d-3p and TMBIM6. Immunohistochemistry was used to measure the expression of TMBIM6. Cell transfection techniques were used to overexpress or interfere with miR-302d-3p and TMBIM6. A Cell Counting Kit-8 assay was performed to detect cell viability, and migration was measured using a wound healing assay. Apoptosis was detected by flow cytometry. The expression levels of apoptosis-related proteins and pathway-related proteins were detected by western blotting. The expression of miR-302d-3p in BC cell lines was found to be downregulated. It was also demonstrated that miR-302d-3p could inhibit cell viability and migration and promote apoptosis. The expression of TMBIM6 in BC cell lines and tissues was upregulated. Upregulated miR-302d-3p was shown to inhibit viability and migration, and promote apoptosis by targeting TMBIM6, during which extracellular signal-regulated kinase (ERK) and its phosphorylation were inhibited in the ERK signaling pathway in cells. Overall, the
\end{abstract}

Correspondence to: Mrs Yanru Liao, Department of Thyroid and Breast Surgery, Baoan Central Hospital of Shenzhen, 60 Xixiang Paradise Street, Baoan, Shenzhen, Guangdong 518102, P.R. China E-mail: liaoyanru2002@163.com

Key words: microRNA-302d-3p, transmembrane Bax inhibitor motif containing 6 , breast cancer, viability, migration, apoptosis, extracellular signal-regulated kinase signaling pathway present study demonstrated that miR-302d-3p could regulate the viability, migration and apoptosis of $\mathrm{BC}$ cells through regulating TMBIM6-mediated ERK signaling pathway.

\section{Introduction}

Breast cancer (BC) is a malignant tumor occurring in the epithelial tissues of the mammary gland. The incidence of $\mathrm{BC}$ worldwide has been on the rise since the late 1970s, with $\sim 1.7$ million new cases detected annually (1). Furthermore, the incidence and mortality of $\mathrm{BC}$ are predicted to increase significantly over the next 5-10 years, along with a decrease in the age of patients with $\mathrm{BC}$ (2). At present, the treatment of $\mathrm{BC}$ mainly comprises surgical resection and postoperative chemotherapy or radiotherapy, but the overall survival rate after treatment is low and the prognosis is poor (3). Therefore, identifying effective targets for the treatment of $\mathrm{BC}$ at the molecular level may significantly improve the survival rates of patients.

Low expression of microRNA (miR/miRNA)-302d-3p has been reported in endometrial cancer cells, while overexpression of miR-302d-3p was revealed to inhibit the epithelial-to-mesenchymal transformation, viability and migration and promote the apoptosis of endometrial cancer cells $(4,5)$. However, it was found that the expression of miR-302d-3p was abnormally increased in the tumor tissues of patients with hepatocellular carcinoma (HCC), and the survival time of patients with $\mathrm{HCC}$ and lower expression of miR-302d was longer compared with that of patients with HCC and higher expression of miR-302d (6). Therefore, the role of miR-302d in cancer varies according to the type of the cancer. Chen and Yang (7) observed that miR-302d was closely associated with the occurrence and development of BC through analyzing microarray data and identifying differentially expressed genes in BC. However, the specific role of miR-302d-3p in BC and its possible underlying mechanism have yet to be reported.

Therefore, the aim of the present study was to investigate the effect of miR-302d-3p on the viability, migration and apoptosis of BC cells and discuss the underlying mechanism, in order to provide a valuable reference for identifying novel therapeutic targets for BC. 


\section{Materials and methods}

BC tissue samples. Between August 2019 and August 2020, a total of 30 pairs of $\mathrm{BC}$ tissue samples and matched adjacent normal tissue (distance, $2 \mathrm{~cm}$ ) were collected from female patients (age range, 20-55 years) who underwent surgery at the Baoan Central Hospital of Shenzhen (Shenzhen, China). All fresh specimens were immediately placed in liquid nitrogen following surgery. All cases were diagnosed using postoperative pathological examination, and other major diseases were excluded. The present study was approved by the Ethics Committee of Baoan Central Hospital of Shenzhen and all patients provided written informed consent. All the procedures complied with the principles outlined in the Declaration of Helsinki and relevant policies in China. Inclusion criteria were as follows: i) Diagnosis by postoperative pathology; ii) imaging examination showed no active lesions; iii) aged 18-65 years; iv) no history of smoking, drinking or recreational drug use; v) provision of signed informed consent. Exclusion criteria were as follows: i) Patients with diabetes or other diseases undergoing treatment; ii) surgery, chemotherapy, nuclear medicine or immunotherapy $<3$ months before enrollment; iii) other malignant tumors; iv) drug treatment $<7$ days before enrollment; v) pregnancy and lactation; vi) blood transfusions $<4$ months before enrollment; vii) hepatitis C, syphilis or HIV antibody-positive.

Cell culture. All cell lines (MCF-10A, MCF7 and MDA-MB-231) were purchased from The Cell Bank of Type Culture Collection of the Chinese Academy of Sciences. The cells were cultured and stored as follows: DMEM (Gibco; Thermo Fisher Scientific, Inc.) was supplemented with $10 \%$ FBS (Gibco; Thermo Fisher Scientific, Inc.) for these cell lines, according to the manufacturer's instructions. All cell lines were maintained in a humidified cell incubator at $37^{\circ} \mathrm{C}$ with an atmosphere of $5 \% \mathrm{CO}_{2}$. ERK pathway inhibitor U0126 $(10 \mathrm{ng} / \mathrm{ml})$ and agonist EGF $(30 \mathrm{ng} / \mathrm{ml}$; both pretreatment for $2 \mathrm{~h}$ at $37^{\circ} \mathrm{C}$; both $98 \%$ pure; both MedChem Express) were used to induce cells.

Reverse transcription-quantitative PCR (RT-qPCR) analysis. The cells were seeded into 6-well plates at a density of $1 \times 10^{6}$ cells/well. Total RNA was extracted using RNA Purified Total RNA Extraction Kit (Invitrogen; Thermo Fisher Scientific, Inc.), and then subjected to reverse transcription to cDNA using SMART MMLC Reverse Transcriptase (Takara Biotechnology Co., Ltd.) at $\sim 65^{\circ} \mathrm{C}$ for $10 \mathrm{~min}$. The qPCR reaction mixture contained $10 \mu 12 \mathrm{X}$ Power Taq PCR MasterMix (cat. no. PR1702; BioTeke Corporation), $0.5 \mu \mathrm{l}$ of each primer [miR-302d-3p forward, 5'-GCGTAAGTGCTTCCATGTTTG TGTGT-3'; transmembrane Bax inhibitor motif containing 6 (TMBIM6) forward, 5'-TCCCTCGACACAGCAGCA CCT-3' and reverse, 5'-CCCCAGAGAGGACAGGAGCAT-3'; GAPDH forward, 5'-AACTTTGGCATTGTGGAAGG-3' and reverse, 5'-GGATGCAGGGATGATGTTCT-3'; and U6 forward, 5'-AACTTTGGCATTGTGGAAGG-3' and reverse, 5'-GGATGCAGGGATGATGTTCT-3'], $1 \mu$ l cDNA template and $8 \mu \mathrm{l}$ RNase free $\mathrm{H}_{2} \mathrm{O}$. GAPDH acted as andogenous control of TMBIM6 and U6 was used as an endogenous control of miR-302d-3p. The thermocycling conditions were as follows: $95^{\circ} \mathrm{C}$ for $10 \mathrm{~min}, 40$ cycles of $95^{\circ} \mathrm{C}$ for $10 \mathrm{sec}$, $55^{\circ} \mathrm{C}$ for $10 \mathrm{sec}$, and $72^{\circ} \mathrm{C}$ for $30 \mathrm{sec}$. The amplification was performed with the Exicycler ${ }^{\mathrm{TM}} 96$ (Bioneer Corporation) and relative expression levels were calculated according to the $2^{-\Delta \Delta \mathrm{Cq}}$ method (8).

Cell Counting Kit-8 (CCK-8) assay. The CCK-8 assay (Dojindo Molecular Technologies, Inc.) was used for the detection of cell viability, according to the manufacturer's protocol. Briefly, cells were seeded in 96-well plates $\left(1 \times 10^{4}\right.$ cells/well) and examined at $24 \mathrm{~h}$. The detection buffer $(100 \mu \mathrm{l}$; ratio of medium to CCK-8, 9:1) was added to each well. After incubation for $3 \mathrm{~h}$ at $37^{\circ} \mathrm{C}$, the absorbance at $450 \mathrm{~nm}$ was detected by a microplate reader.

Wound healing assay. Cells were seeded at 6-well plates ( $1 \times 10^{6}$ cells/well)and grown to $80 \%$ confluence in 12 -well plates. Two linear scratches were created in the cell monolayer with a $200-\mu 1$ micropipette tip in each well, after which time the cells were treated in serum-free media and allowed to migrate for $24 \mathrm{~h}$. Images of the wound areas were captured under an AxioVert 200M fluorescence microscope (magnification, x100; Carl Zeiss AG) at 0 and 24 h. Migration was calculated according to the following formula: Cell mobility $=(0 \mathrm{~h}$ scratch width-scratch width after culture)/0 h scratch width.

TUNEL assay. The cells were collected and washed with PBS three times. Cells were fixed with $4 \%$ paraformaldehyde for $30 \mathrm{~min}$ at room temperature and washed with PBS. Then, $0.3 \%$ Triton X-100 in PBS was added and incubated for $5 \mathrm{~min}$ at room temperature. Subsequently, $50 \mu 1$ TUNEL assay solution (Roche Diagnostics $\mathrm{GmbH}$ ) was added to the cells and incubated at $37^{\circ} \mathrm{C}$ in the dark for $60 \mathrm{~min}$. Next, cells were incubated with DAB and stained with hematoxylin and eosin for $5 \mathrm{~min}$ at room temperature. The detection solution was then discarded and the cells were washed with PBS three times and sealed with anti-fluorescence quenched sealing solution. A total of 3 visual fields were randomly selected for observation under a fluorescence microscope (magnification, x200; Carl Zeiss AG). The available excitation wavelength range was 450-500 $\mathrm{nm}$ and the emission wavelength range was 515-565 $\mathrm{nm}$ (green fluorescence).

Western blot analysis. The samples were treated with RIPA lysis buffer (Beyotime Institute of Biotechnology), incubated on ice for $30 \mathrm{~min}$ and later centrifuged at $300 \mathrm{x} \mathrm{g}$ for $20 \mathrm{~min}\left(4^{\circ} \mathrm{C}\right)$. The protein concentration was determined using a bicinchoninic acid assay protein assay kit (Beyotime Institute of Biotechnology). Total protein $(30 \mu \mathrm{g})$ was collected and separated via 10\% SDS-PAGE, and subsequently transferred to PVDF membranes and blocked in $5 \%$ non-fat milk at room temperature for $1 \mathrm{~h}$. The membranes were incubated overnight at $4^{\circ} \mathrm{C}$ with polyclonal rabbit anti-Bcl-2 antibody (1:1,000; cat. no. ab32124; Abcam), rabbit anti-Bax antibody (1:1,000; cat. no. ab32503; Abcam), anti-caspase-3 antibody (1:1,000; cat. no. ab197202; Abcam), anti-cleaved caspase-3 (1:1,000; cat. no. PA5-17913; Thermo Fisher Scientific, Inc.), anti-caspase-9 antibody (1:1,000; cat. no. ab219590; Abcam), anti-cleaved caspase-9 (1:1,000; cat. no. PA5-17605; Thermo Fisher Scientific, Inc.), 
anti-extracellular signal-regulated kinase (ERK) antibody (1:1,000; cat. no. ab32537; Abcam), anti-phosphorylated (p)-ERK antibody (1:1,000; cat. no. ab192591; Abcam) and anti- $\beta$-actin antibody (1:1,000; cat. no. ab179467; Abcam), followed by incubation with HRP-labeled goat anti-rabbit secondary antibody (1:5,000; cat. no. A-11012; Thermo Fisher Scientific, Inc.) for $1 \mathrm{~h}$ at room temperature. Proteins were visualized using ImageQuant ${ }^{\mathrm{TM}}$ LAS 4000 (Cytiva) and semi-quantified using ImageJ software (version 1.46; National Institutes of Health).

Dual-luciferase reporter assay. Bioinformatics software TargetScan (http://www.targetscan.org) was used to predict the target genes of miR-302d-3p, and TMBIM6 was determined as a potential target. The wild-type (WT) or mutant (MUT) miR-302d-3p-binding site was subcloned into a pCDNA3.1 plasmid purchased from Thermo Fisher Scientific, Inc. The cells were plated in the 24 -well plates ( $1 \times 10^{4}$ cells/well) $24 \mathrm{~h}$ before transfection. miR-302d-3p-mimic, miR-302d-3p-inhibitor and corresponding controls were co-transfected with $10 \mu \mathrm{g}$ pCDNA3.1-WT-miR-302d-3p or pCDNA3.1-MUT-miR-302d-3p for $48 \mathrm{~h}$ at $37^{\circ} \mathrm{C}$ using Lipofectamine ${ }^{\circledR} 2000$ (Thermo Fisher Scientific, Inc.) according to the manufacturer's protocol. The luciferase activity was measured using a plate reader (BD Biosciences) and was normalized to Renilla luciferase activity (pRL-TK) using the Luc-Screen ${ }^{\mathrm{TM}}$ Extended-Glow Luciferase Reporter Gene Assay system (cat. no. E1910; Promega Corporation).

Immunohistochemical analyses. Tissue samples $(0.2 \mathrm{~cm})$ were fixed in $10 \%$ formalin for $24 \mathrm{~h}$ at room temperature, embedded in paraffin, and analyzed by immunohistochemistry. The samples were blocked in 5\% normal goat serum (Gibco; Thermo Fisher Scientific, Inc.) for $1 \mathrm{~h}$ at room temperature, probed with anti-TMBIM6 (1:150; cat. no. ab18852; Abcam) at $4^{\circ} \mathrm{C}$ overnight and labeled with biotinylated secondary antibodies $\left(1: 2,000\right.$; cat. no. ab205718; Abcam) for $1 \mathrm{~h}$ at $37^{\circ} \mathrm{C}$. The immunoreaction signal was developed with DAB staining, and the slides were counterstained in hematoxylin for $5 \mathrm{~min}$ at room temperature. Stained tissue sections were viewed under a light microscope (magnification, x200; ECLIPSE Ni-U; Nikon Corporation). Histological score (H-score) was used to calculate staining score as follows: $\mathrm{H}$-score=intensity $\mathrm{x}$ total number of positive cells x $100 \%$.

Cell transfection. The mimic and the inhibitor of hsa-miR302d-3p, miRNA negative controls (miR-302d-3p-NC), TMBIM6-smalll interfering (si)RNA and negative control vector (TMBIM6-NC) at a concentration of $20 \mathrm{nM}$ were synthesized by Shanghai GenePharma Co., Ltd. Cells at a final concentration of $25 \mathrm{nM}\left(1 \times 10^{6}\right.$ cells/well $)$ were transfected using Lipofectamine ${ }^{\circledR} 3000$ reagent (Invitrogen; Thermo Fisher Scientific, Inc.) according to the manufacturer's protocol. Following incubation for $48 \mathrm{~h}$ at $37^{\circ} \mathrm{C}$, cells were used for subsequent experiments. Transfection efficiency was detected via RT-qPCR. The sequences of mimics/inhibitors/siRNAs were as follows: NC-mimic, 5'-UUCUCCGAACGUGUCACGUTT-3'; NC-inhibitor, 5'-CAGUACUUUUGUGUAGUACAA-3'; miR-302d-3p mimic, 5'-UAAGUGCUUCCAUGU UUGAGUGU-3';
miR-302d-3p inhibitor, 5'-ACACUCAAACAUGGAAGC ACUUA-3'; si-TMBIM6 forward, 5'-GUGGAAGGCCUU CUUUCUA-3' and reverse, 5'-UAGAAAGAAGGCCUU CCAC-3'; and TMBIM6-NC forward, 5'-CUGAACAAC CAAUGCAAAU-3' and reverse, 5'-AUUUGCAUUGGU UGUUCAG-3'. The cells were grouped as follows: Control, miR-302d-3p-NC, TMBIM6-NC, miR-302d-3p mimics, miR-302d-3p mimics + si-TMBIM6, miR-302d-3p inhibitor, miR-302d-3p inhibitor + si-TMBIM6 and si-TMBIM6. Subsequently, the cells were divided into control, miR-302d-3p-NC, miR-302d-3p mimics, miR-302d-3p mimics + U0126, miR-302d-3p mimics + EGF, miR-302d-3p inhibitor, miR-302d-3p inhibitor + U0126 and miR-302d-3p inhibitor + EGF groups.

Statistical analysis. All data are presented as the mean \pm standard deviation. Multiple comparisons among three groups were performed using one-way ANOVA followed by Tukey's post hoc test. Error bars represent standard deviation. $\mathrm{P}<0.05$ was considered to indicate a statistically significant difference.

\section{Results}

miR-302d-3p inhibits viability and migration and promotes apoptosis of BC cells. The expression of miR-302d-3p in the BC cell lines MCF7 and MDA-MB-231 was significantly downregulated compared with normal breast epithelial cells, as determined via RT-qPCR (Fig. 1A). The decrease in miR-302d-3p expression levels was greatest in MCF-7 cells, therefore MCF7 cells were selected for subsequent experiments. miR-302d-3p mimics, miR-302d-3p inhibitor and miR-302d-3p-NC were transfected into MCF7 BC cells. The transfection efficiency was confirmed by RT-qPCR analysis (Fig. 1B). Subsequently, the CCK-8 assay demonstrated that, compared with the miR-302d-3p-NC, the cell survival rate of the miR-302d-3p mimics group was decreased, whereas that of the miR-302d-3p inhibitor group was increased (Fig. 1C). The wound healing assay also demonstrated that the cell migration rate of the miR-302d-3p mimics group was decreased, whereas that of the miR-302d-3p inhibitor group was increased (Fig. 1D and E). The TUNEL assay revealed increased apoptosis in the miR-302d-3p mimics group, whereas no apoptosis was observed in the miR-302d-3p inhibitor group (Fig. 2A). Western blot analysis of the expression levels of the apoptosis-related proteins Bcl-2, Bax, caspase-3 and caspase-9 revealed a consistent trend. Compared with miR-302d-3p-NC group, Bcl-2 was decreased and Bax, caspase-3 and caspase-9 were increased in miR-302d-3p mimic group. Compared with miR-302d-3p-NC group, Bcl-2 was increased and Bax, caspase-3 and caspase- 9 were decreased in miR-302d-3p inhibitor group (Fig. 2B). The aforementioned results indicated that miR-302d-3p may inhibit the viability and migration, and promote the apoptosis, of $\mathrm{BC}$ cells.

miR-302d-3p inhibits the viability and migration of $B C$ cells and promotes apoptosis by targeting the expression of TMBIM6. The binding site of miR-302d-3p and the 3'-untranslated region (UTR) of TMBIM6 were predicted using TargetScan (Fig. 3A) and the binding site of miR-302d-3p and TMBIM6 was verified with a dual-luciferase reporter assay 
A
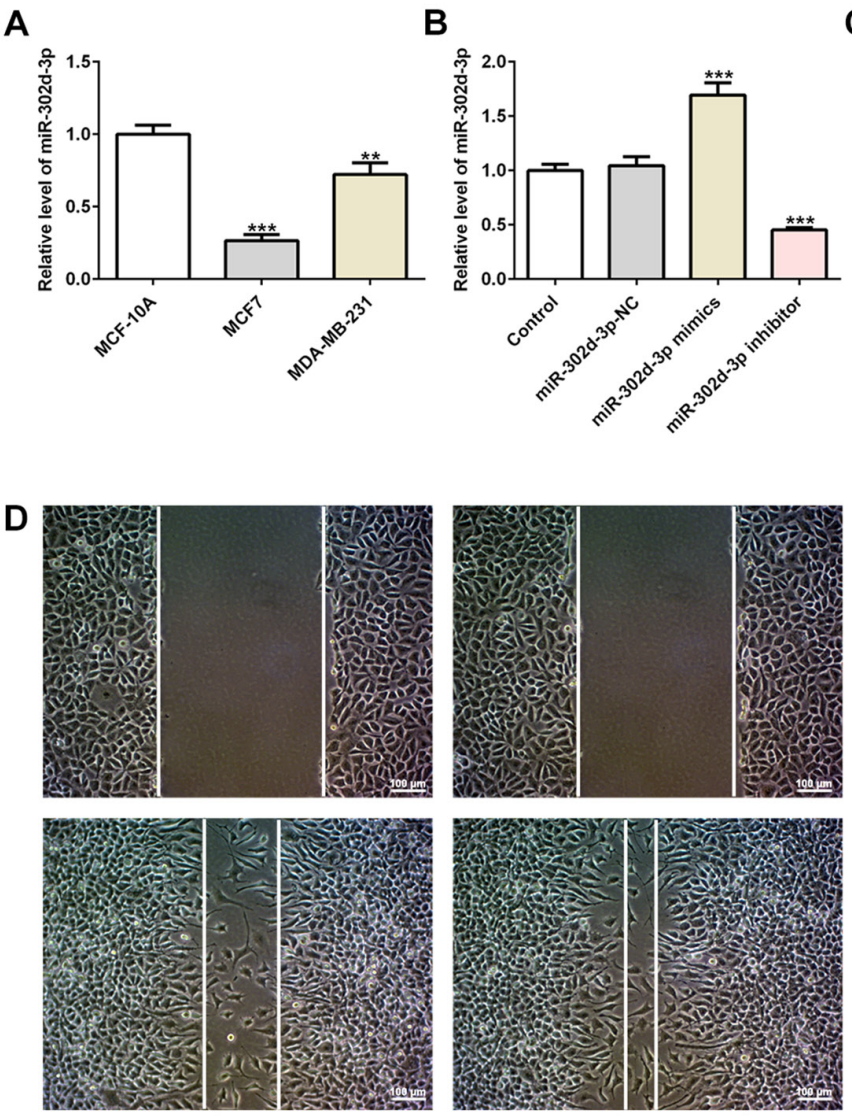

Control
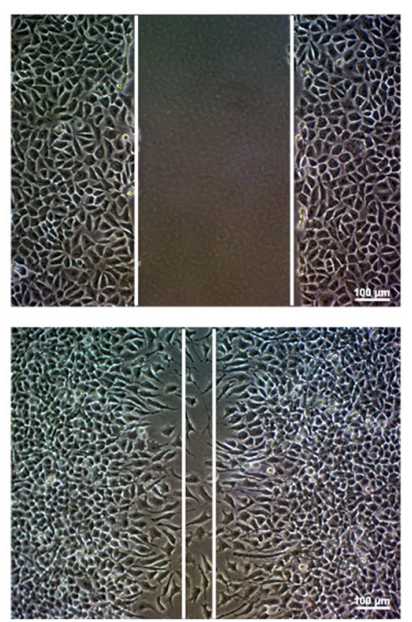

miR-302d-3p-NC
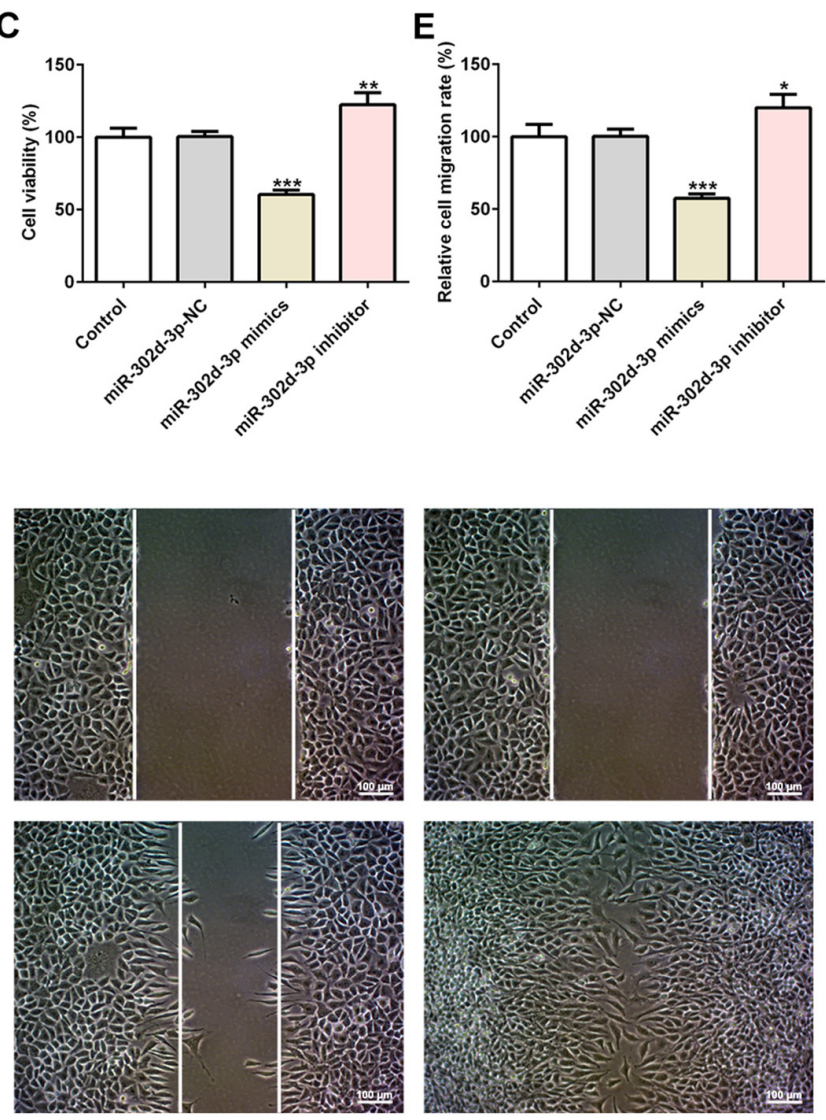

miR-302d-3p mimics

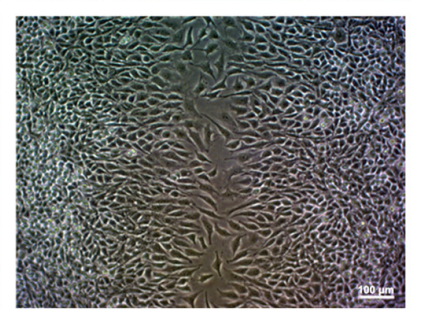

miR-302d-3p inhibitor

Figure 1. miR-302d-3p inhibits viability and migration of BC cells. (A) RT-qPCR was performed to detect the expression of miR-302d-3p in BC cell lines. (B) RT-qPCR was used to measure the expression of miR-302d-3p in cells following transfection. (C) Cell Counting Kit- 8 assay was performed to detect cell viability. ( $\mathrm{D}$ and $\mathrm{E}$ ) Wound healing assay was used to detect cell migration (scale bar, $100 \mu \mathrm{m}$ ). ${ }^{*} \mathrm{P}<0.05,{ }^{* *} \mathrm{P}<0.01,{ }^{* * *} \mathrm{P}<0.001 \mathrm{vs}$. miR-302d-3p-NC. miR, microRNA; BC, breast cancer; RT-qPCR, reverse transcription-quantitative PCR; NC, negative control.

(Fig. 3B). Subsequently, immunohistochemistry was used to detect the expression of TMBIM6 in BC samples and normal adjacent tissues, and it was observed that the expression of TMBIM6 in BC tissues was increased (Fig. 3C and D). In addition, RT-qPCR assays were performed to detect the expression of TMBIM6 in the serum of 30 samples from patients with BC (Fig. 3E) and in BC cell lines (Fig. 3F), which were both consistent with the results presented in Fig. 3D.

In order to further test the role of TMBIM6 in the viability, migration and apoptosis of BC cells, miR-302d-3p mimics, miR-302d-3p inhibitor, si-TMBIM6 and the corresponding NC groups miR-302d-3p-NC and TMBIM6-NC were synthesized and transfected into MCF7 cells. It was observed that the expression of TMBIM6 decreased when miR-302d-3p was overexpressed and increased when miR-302d-3p was inhibited compared with miR-302d-3p-NC (Fig. 4A). Compared with miR-302d-3p-NC, expression of miR-302d-3p increased in miR-302d-3p mimic, whereas expression of miR-302d-3p decreased in miR-302d-3p inhibitor group (Fig. 4B). This finding further indicated that miR-302d-3p may target TMBIM6. As shown in Fig. 4C, cell viability significantly decreased when cells were transfected with miR-302d-3p mimics compared with miR-302d-3p-NC, and a further decrease in the cell survival rate was observed following transfection with si-TMBIM6 compared with miR-302d-3p mimics. Cell viability was increased in the miR-302d-3p inhibitor group compared with that of the miR-302d-3p-NC group, and further interference with TMBIM6 resulted in a significant decrease in cell viability. Compared with the TMBIM6-NC group, the cell viability of the si-TMBIM6 group decreased. The wound healing assay revealed that, compared with the si-TMBIM6 group, cell migration exhibited a decreasing trend in the miR-302d-3p mimics + si-TMBIM6 group, whereas cell migration increased in the miR-302d-3p inhibitor + si-TMBIM6 group. Moreover, the rate of cell migration was always decreased following transfection with si-TMBIM6 compared with miR-302d-3p mimics, despite miR-302d-3p knockdown or overexpression (Fig. 4D and E). The results revealed that, compared with TMBIM6-NC, the si-TMBIM6 group had fewer apoptotic cells. After knockdown or overexpression of miR-302d-3p, the rate of apoptosis was notably increased after further interference with si-TMBIM6 (Fig. 5A). Next, western blotting was used to detect the expression of apoptosis-related proteins (Fig. 5B and C), and the findings were consistent with those shown in Fig. 5A. These results suggested that miR-302d-3p inhibited viability and migration, and promoted apoptosis of BC cells by targeting the expression of TMBIM6.

miR-302d-3p regulates the ERK signaling pathway via targeting TMBIM6 to inhibit viability and migration and promote apoptosis of BC cells. In this experiment, changes were 

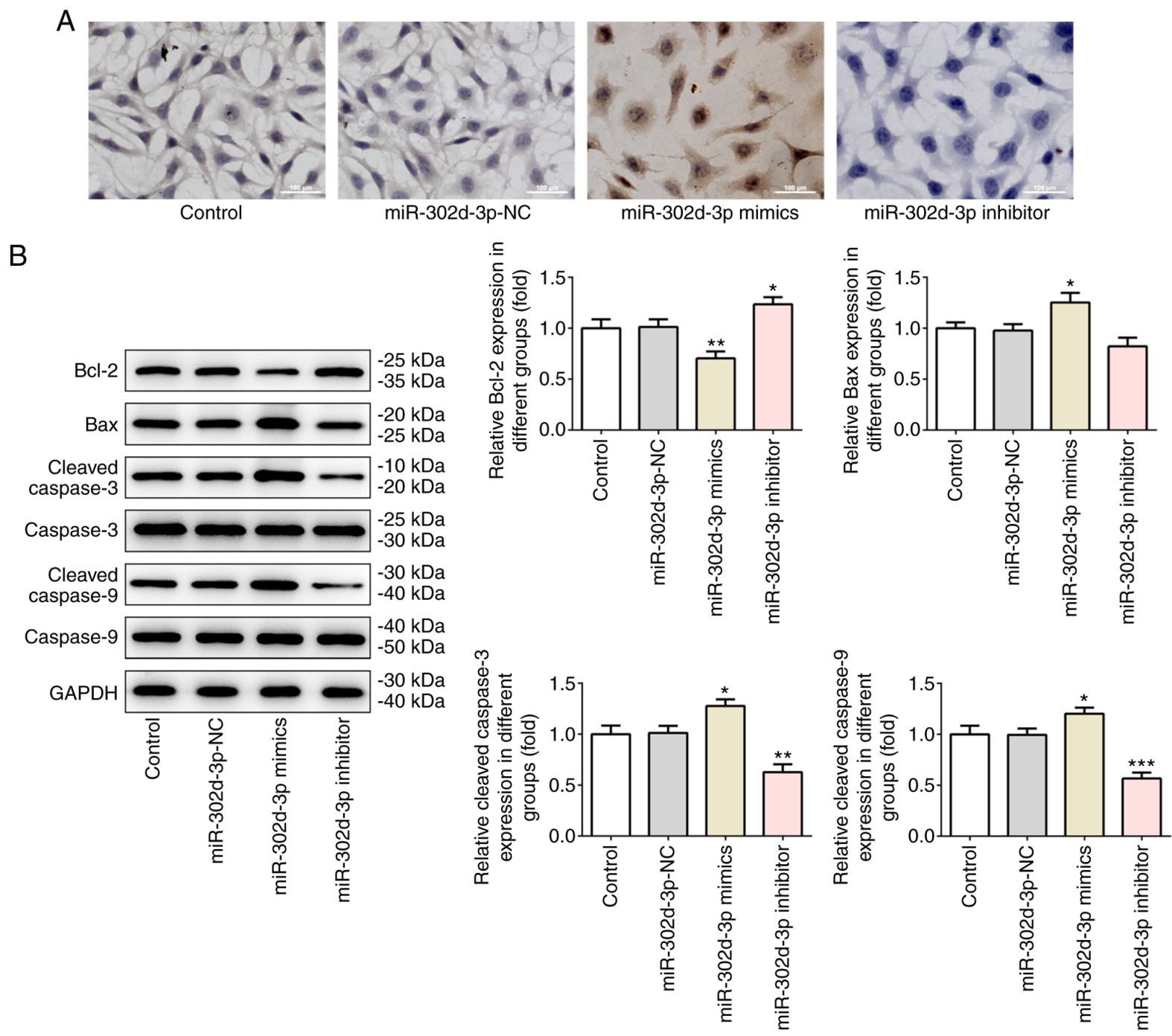

Figure 2. miR-302d-3p promotes apoptosis of BC cells. (A) TUNEL assay was performed to detect the apoptosis rate of BC cells (scale bar, $100 \mu \mathrm{m}$ ). (B) Western blotting was used to determine the expression of apoptosis-related proteins. ${ }^{*} \mathrm{P}<0.05,{ }^{* *} \mathrm{P}<0.01,{ }^{* * *} \mathrm{P}<0.001$ vs. miR-302d-3p-NC. miR, microRNA; $\mathrm{BC}$, breast cancer; NC, negative control.

also found in the expression of ERK pathway-related proteins. It was observed that, compared with the miR-302d-3p-NC group, the expression of $\mathrm{p}$-ERK was decreased following overexpression of miR-302d-3p (Fig. 6A), whereas p-ERK expression was significantly increased after knockdown of miR-302d-3p expression (Fig. 6B). Compared with TMBIM6-NC, the expression of p-ERK significantly decreased after transfection with si-TMBIM6. Compared with miR-302d-3p mimics, the expression of p-ERK was further decreased after knockdown of TMBIM6. Compared with miR-302d-3p inhibitor, the expression of $\mathrm{p}$-ERK decreased after transfection with si-TMBIM6. These results suggested that miR-302d-3p inhibited viability and migration and promoted apoptosis of BC cells via targeting TMBIM6, which may be related to the ERK signaling pathway. Therefore, in the following experiments, an inhibitor of the ERK pathway, U0126, and an EGF agonist were added after cell transfection to further test the underlying mechanism. After overexpression or knockdown of miR-302d-3p through cell transfection, there was no significant change in the expression of the ERK protein, whereas the expression of p-ERK was altered following the addition of
U0126 or EGF. Compared with the miR-302d-3p-NC group, the expression of p-ERK decreased after overexpression of miR-302d-3p. Compared with the miR-302d-3p mimics group, the expression of $\mathrm{p}$-ERK was further inhibited after the addition of U0126, and the decreasing trend in p-ERK expression was reversed following the addition of EGF (Fig. 7A). Compared with the miR-302d-3p-NC group, the expression of p-ERK increased after the inhibition of miR-302d-3p. This increase in p-ERK was reversed following the addition of U0126, whereas the expression of p-ERK was enhanced after the addition of EGF, compared with the miR-302d-3p inhibition group (Fig. 7B).

Subsequently, it was observed that, compared with miR-302d-3p mimics and miR-302d-3p inhibitor, the addition of U0126 reduced cell viability (Fig. 8A) and migration (Fig. 8B and C), and notably increased apoptosis (Fig. 9A). The addition of EGF promoted an increase in cell viability and cell migration, and cell apoptosis was markedly decreased. Subsequently, the expression levels of apoptosis-related proteins were detected by western blotting. The results demonstrated that, compared with miR-302d-3p-NC, the expression 
A

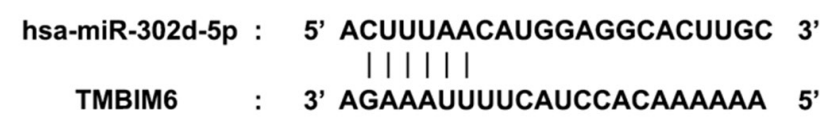

C

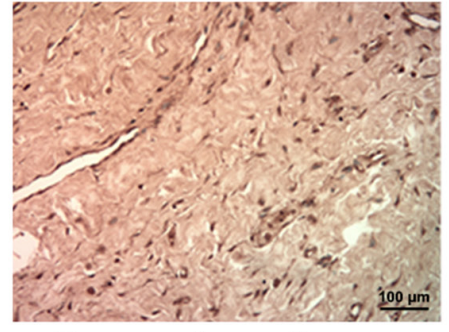

Para-carcinoma tissue

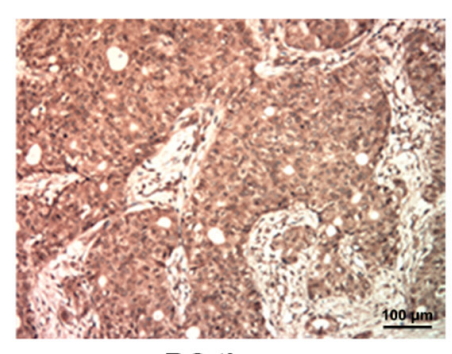

BC tissue

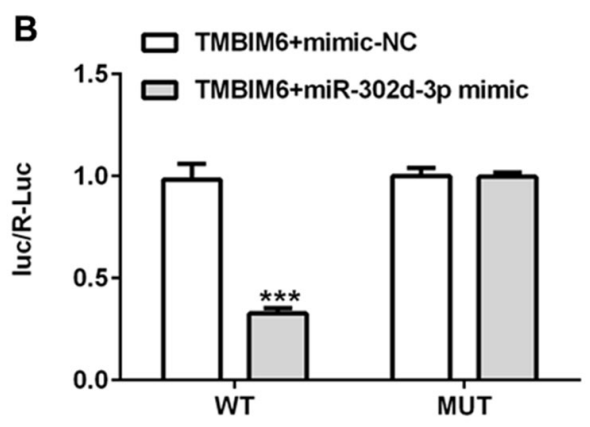

D

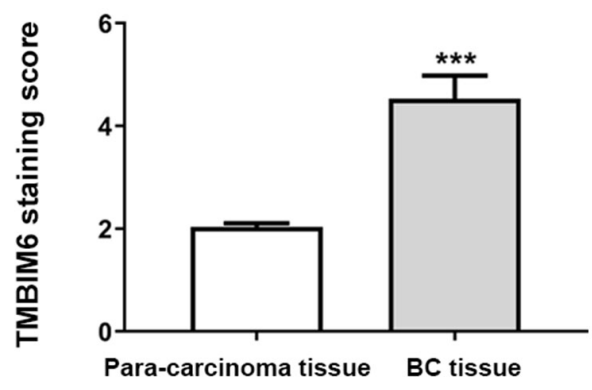

$\mathbf{F}$

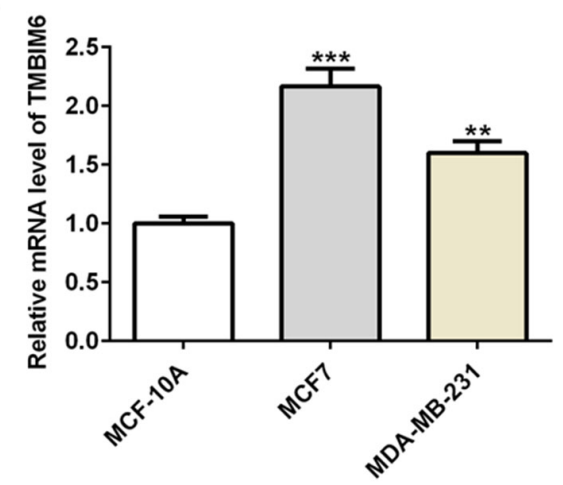

Figure 3. Expression of TMBIM6 in BC. (A) Binding sequence of miR-302d-3p and TMBIM6. (B) Dual-luciferase reporter assay was performed to verify the binding site between miR-302d-3p and TMBIM6. ${ }^{* * *} \mathrm{P}<0.001$ vs. TMBIM6 + mimic-NC. (C) Immunohistochemistry was used to detect TMBIM6 expression in patients with $\mathrm{BC}$ (scale bar, $100 \mu \mathrm{m}$ ). ${ }^{* * * *} \mathrm{P}<0.001$. (D) Statistical analysis of immunohistochemistry score. (E) RT-qPCR was performed to detect the expression of TMBIM6 in patients with $\mathrm{BC}$. $^{* * *} \mathrm{P}<0.001$ vs. para-carcinoma tissues. (F) RT-qPCR was used to detect the expression of TMBIM6 in BC cells. ${ }^{* *} \mathrm{P}<0.01$, ${ }^{* * * *} \mathrm{P}<0.001$ vs. MCF-10A cells. miR, microRNA; BC, breast cancer; RT-qPCR, reverse transcription-quantitative PCR; NC, negative control; TMBIM6, transmembrane Bax inhibitor motif containing 6.

of Bcl-2 was decreased and the expression levels of Bax and caspase-3/9 were increased following overexpression of miR-302d-3p. Furthermore, after the addition of U0126, the expression of $\mathrm{Bcl}-2$ was further decreased, whereas the expression levels of Bax and caspase $3 / 9$ were further increased, compared with the miR-302d-3p mimics group. Following the addition of EGF, the changes in the expression of all proteins were the opposite of those induced by U0126 (Fig. 9B). Compared with miR-302d-3p-NC, expression of Bcl-2 increased, and the expression levels of Bax and caspase-3/9 decreased following inhibition of miR-302d-3p. Following the addition of U0126, compared with the miR-302d-3p inhibition group, the expression of Bcl-2 was decreased, and the expression levels of Bax and caspase-3/9 were increased, which were both reversed after the addition of EGF (Fig. 9C). Therefore, these results indicated that $\mathrm{miR}-302 \mathrm{~d}-3 \mathrm{p}$ regulates the ERK signaling pathway via targeting TMBIM6 to inhibit viability and migration and promote apoptosis of BC cells.

\section{Discussion}

miR-302d-3p is a recently identified cancer-regulating gene that plays a key role in cancer development (9). miR-302d may bind to the 3'-UTR of cyclin D1 mRNA, thus inhibiting the viability of bladder cancer cells (10). The expression of miR-302d was found to be downregulated in human glioblastoma cells, and miR-302d inhibited the occurrence of tumors in xenograft model mice (11). However, the effect of miR-302d-3p on the viability, migration and apoptosis of $\mathrm{BC}$ cells and its underlying mechanism have not been reported to date. The present study demonstrated that the expression of miR-302d-3p in $\mathrm{BC}$ cell lines was decreased. Subsequently, the expression of miR-302d-3p was promoted or inhibited through cell transfection, and it was observed that miR-302d-3p inhibited viability and migration, and promoted apoptosis of BC cells.

Using TargetScan bioinformatics software, it was predicted that the 3'-UTR region of TMBIM6 had binding sites for 
A

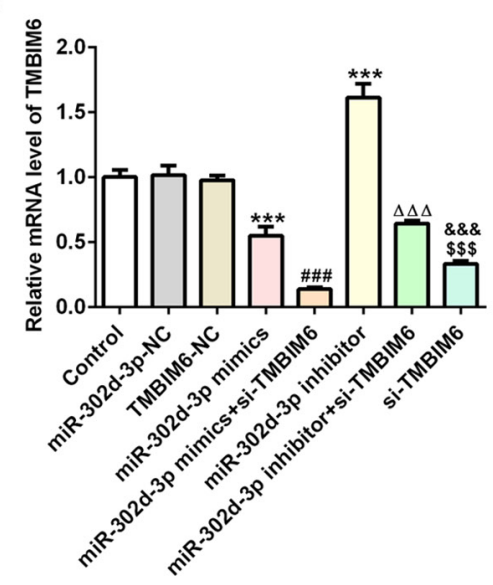

B

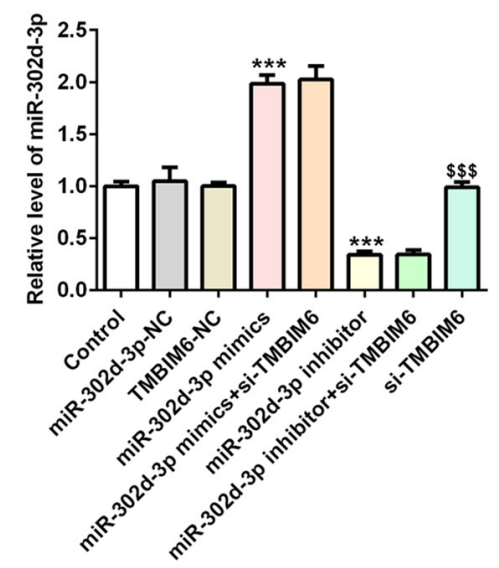

C

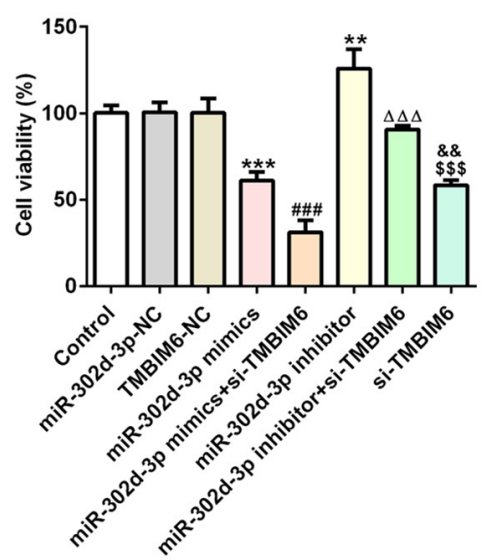

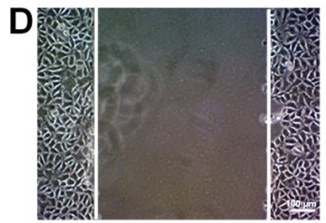
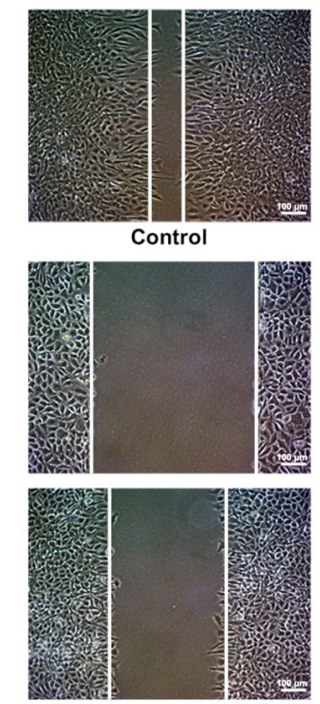
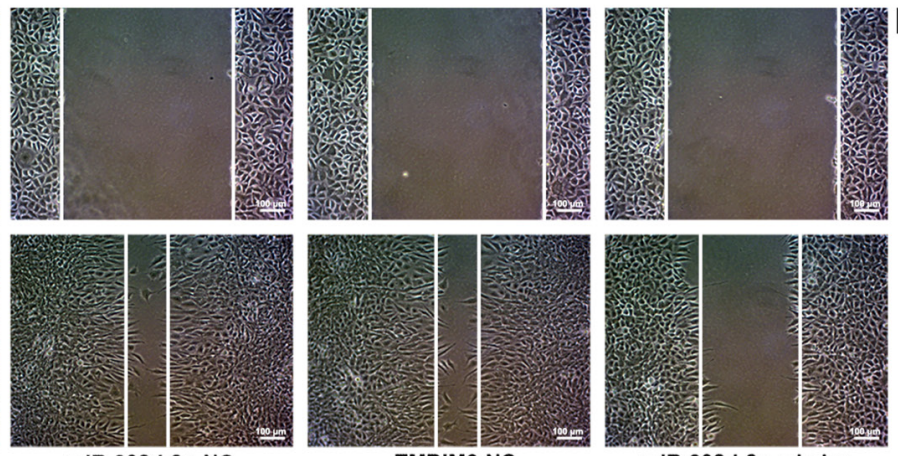

miR-302d-3p-NC
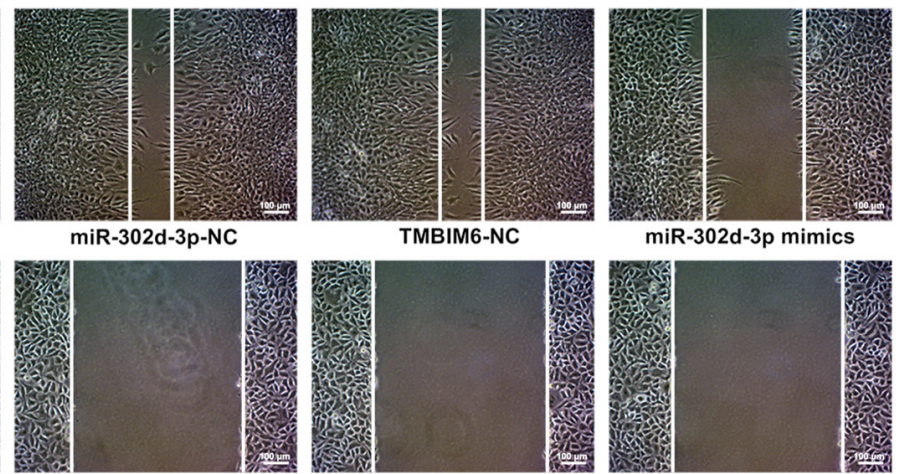

miR-302d-3p mimics+si-TMBIM6 miR-302d-3p inhibitor miR-302d-3p inhibitor+si-TMBIM6
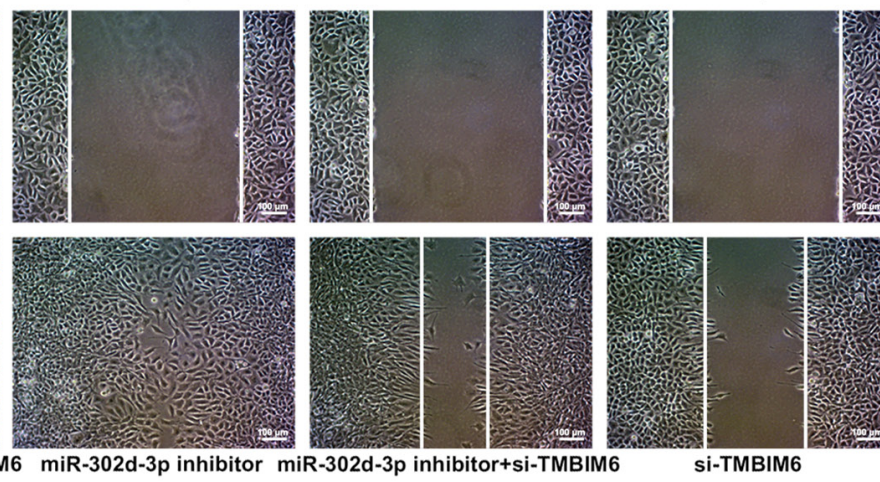

riving

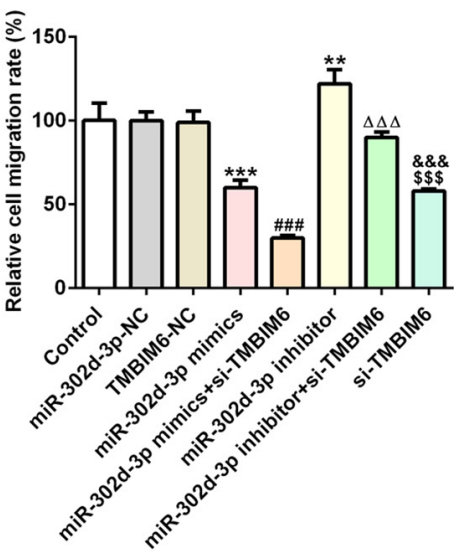

Figure 4. miR-302d-3p inhibits the viability and migration of BC cells by targeting the expression of TMBIM6. (A) RT-qPCR was performed to detect the expression of TMBIM6 in cells following cell transfection. (B) RT-qPCR was used to determine the expression of miR-302d-3p in cells following cell transfection. (C) Cell Counting Kit-8 was performed to measure cell viability. (D and E) A wound healing assay was conducted to detect cell migration

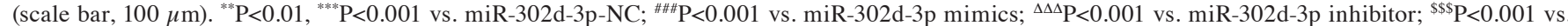
TMBIM6-NC. \&\& $\mathrm{P}<0.01$ and ${ }^{\& \& \&} \mathrm{P}<0.001$ vs. miR-302d-3p inhibitor+si-TMBIM6. miR, microRNA; BC, breast cancer; RT-qPCR, reverse transcription-quantitative PCR; NC, negative control; TMBIM6, transmembrane Bax inhibitor motif containing 6; si-, small interfering RNA.

miR-302d-3p. TMBIM6, also referred to as Bax inhibitor-1, is an evolutionarily conserved transmembrane protein that is primarily located in the endoplasmic reticulum (12). TMBIM6 has been identified as an anti-apoptotic protein due to its protective effect against the pro-apoptotic protein Bax (13). TMBIM6 is upregulated in a number of tumors and is involved in metastasis, whereas the downregulation of TMBIM6 may lead to cancer cell death (14). It has been reported that TMBIM6 is highly expressed in the cells and serum of patients with BC (15). In the present study, the targeting association between miR-302d-3p and TMBIM6 was first verified through a dual-luciferase reporter assay. Moreover, the expression of TMBIM6 was found to be significantly increased in BC tissues and cell lines. Next, miR-302d-3p overexpression and interference with the expression of miR-302d-3p and TMBIM6 was achieved through cell transfection, and cell viability, migration and apoptosis were detected. The findings demonstrated that knockdown of TMBIM6 could further inhibit cell viability and migration and further promote apoptosis induced by miR-302d-3p.

ERK is an important member of the mitogen-activated protein kinase (MAPK) family and plays an important role in regulating different biological processes in different cells, including proliferation, differentiation, survival and apoptosis (16). The results of the present study indicated that the activation of the ERK signaling pathway exerted anti-apoptotic 
A

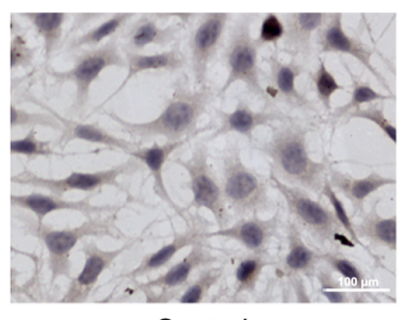

Control

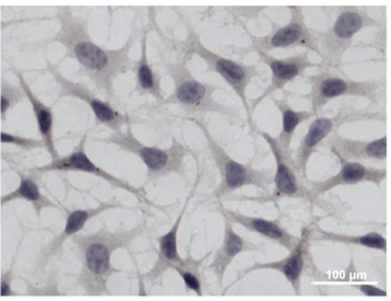

miR-302d-3p-NC

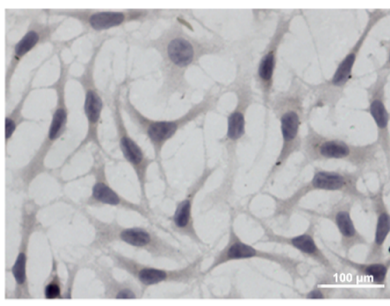

TMBIM6-NC
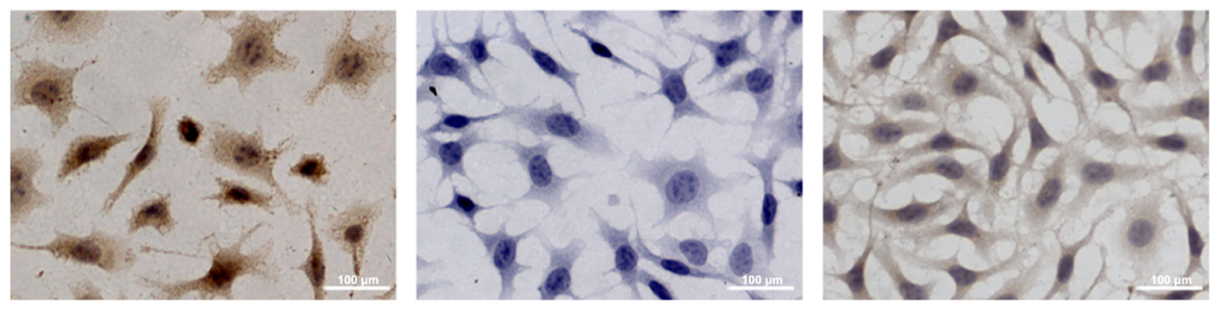

miR-302d-3p inhibitor+si-TMBIM6

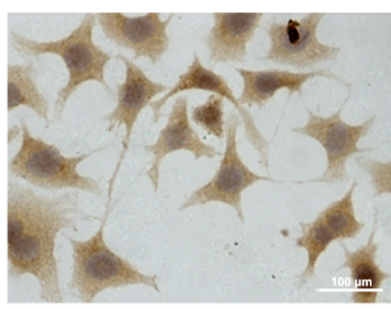

miR-302d-3p mimics

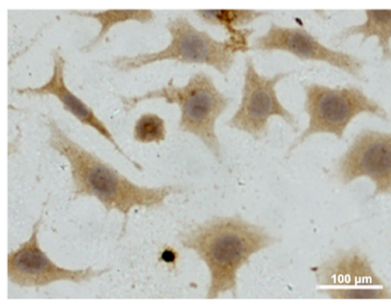

si-TMBIM6
B

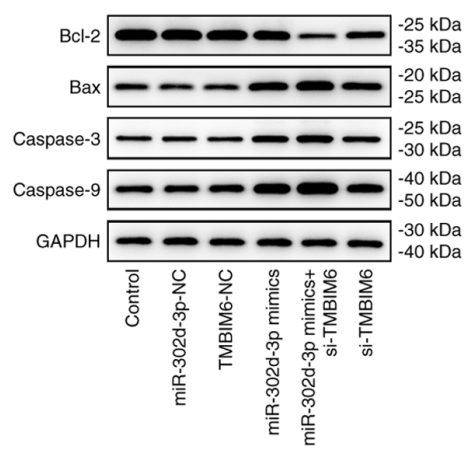

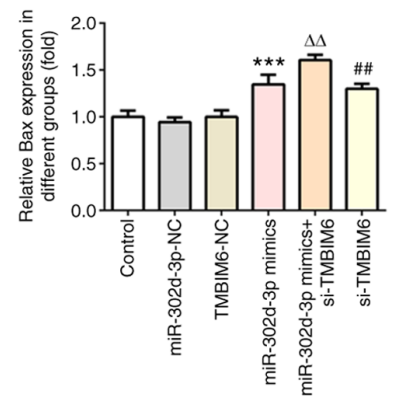

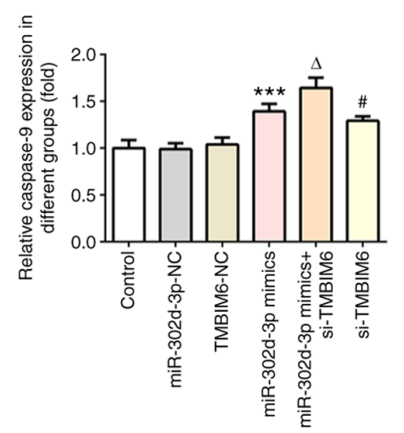

C

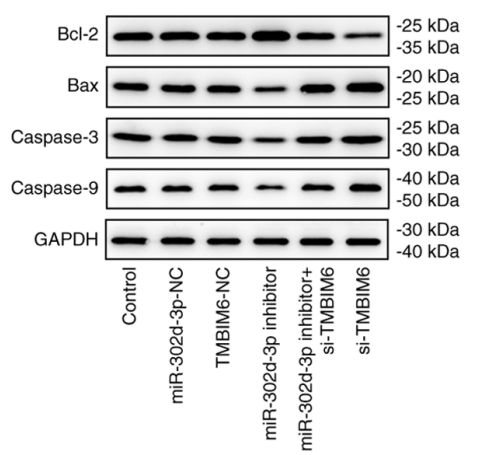

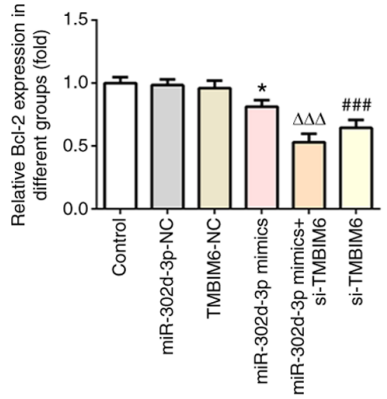

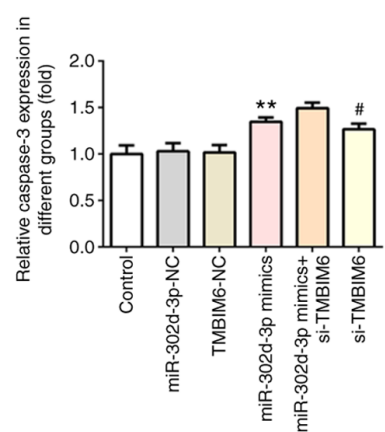

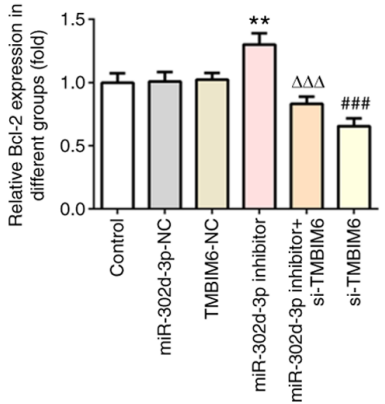
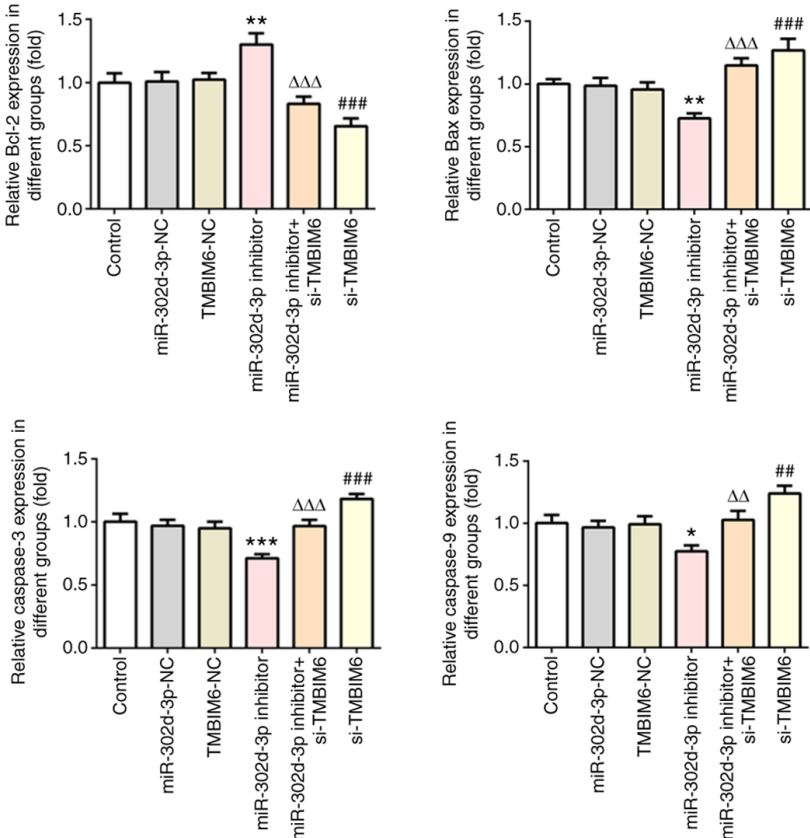

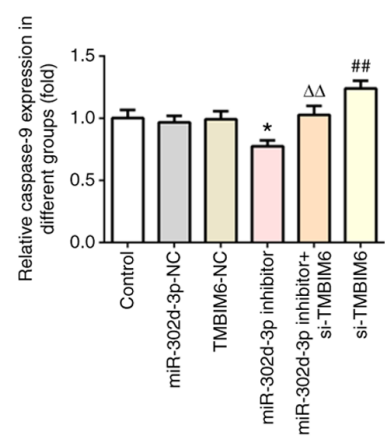

Figure 5. miR-302d-3p promotes apoptosis by targeting TMBIM6. (A) TUNEL assay was performed to determine the apoptosis rate of cells following transfection (scale bar, $100 \mu \mathrm{m}$ ). (B and C) Western blotting was performed to detect the expression of apoptosis-related proteins. ${ }^{*} \mathrm{P}<0.05,{ }^{* *} \mathrm{P}<0.01,{ }^{* * *} \mathrm{P}<0.001$ vs. miR-302d-3p-NC; ${ }^{\Delta} \mathrm{P}<0.05,{ }^{\Delta \Delta} \mathrm{P}<0.01,{ }^{\Delta \Delta \Delta} \mathrm{P}<0.001$ vs. miR-302d-3p mimics or inhibitor; ${ }^{\# P} \mathrm{P}<0.05,{ }^{\# \#} \mathrm{P}<0.01,{ }^{\# \# "} \mathrm{P}<0.001$ vs. TMBIM6-NC. miR, microRNA; NC, negative control; TMBIM6, transmembrane Bax inhibitor motif containing 6; si-, small interfering RNA.

effects on cancer cells, which could therefore promote the occurrence and development of BC, as commonly observed in other types of cancer, including pancreatic cancer, esophageal squamous cell carcinoma and thyroid cancer (17-20).
Zhao et al (21) investigated the sensitivity of $\mathrm{BC}$ cells to Adriamycin and found that miR-302d regulated cell sensitivity to Adriamycin by regulating MAPK/ERK kinase kinase 1 to inhibit the expression of p-glycoprotein. Therefore, the 
A

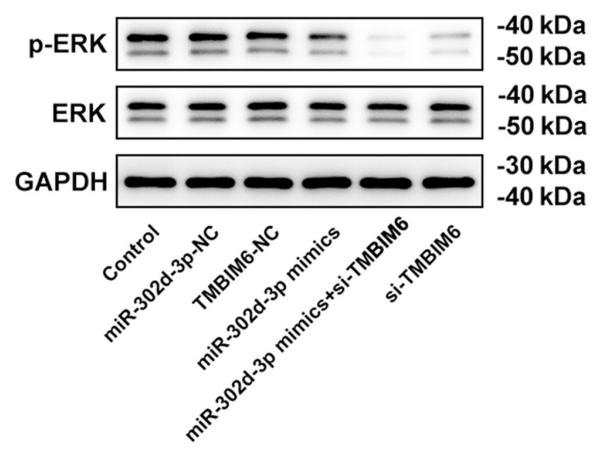

B

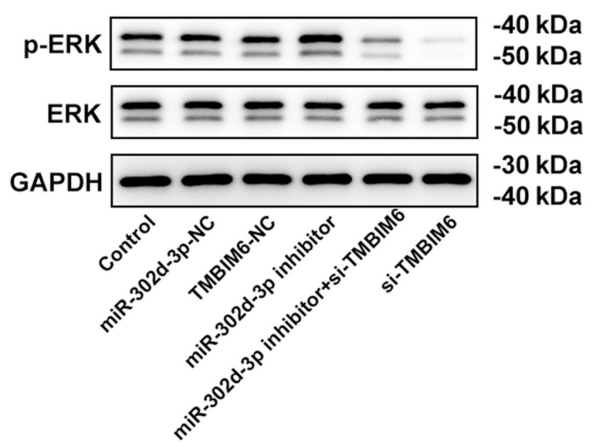

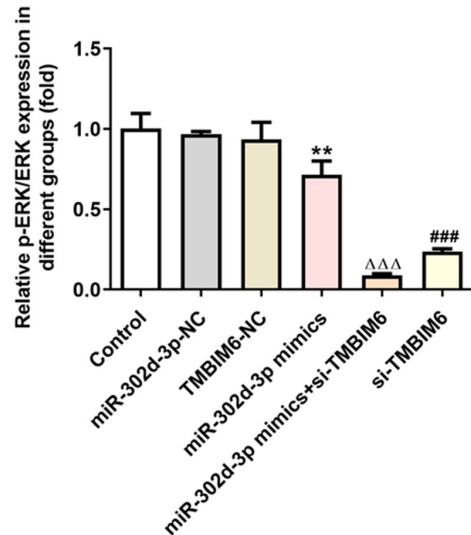

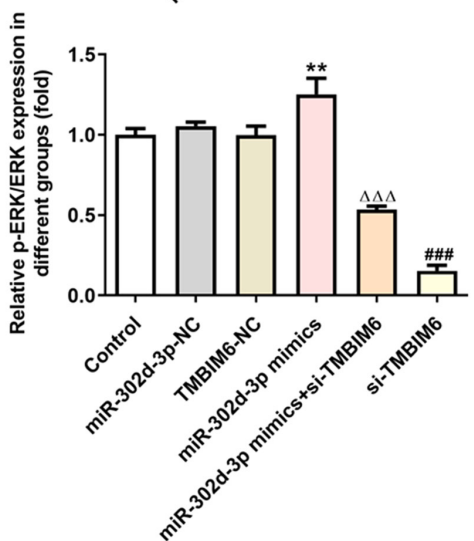

Figure 6. Expression of ERK pathway-related proteins. Western blotting was performed to detect the expression of ERK pathway-related proteins following transfection with (A) miR-302d-3p mimics or (B) miR-302d-3p inhibitor plus si-TMBIM6. ${ }^{* *} \mathrm{P}<0.01$ vs. miR-302d-3p-NC; ${ }^{\Delta \Delta \Delta} \mathrm{P}<0.001 \mathrm{vs}$. miR-302d-3p mimics or inhibitor; ${ }^{\# \# / ~} \mathrm{P}<0.001$ vs. TMBIM6-NC. miR, microRNA; NC, negative control; TMBIM6, transmembrane Bax inhibitor motif containing 6; si-, small interfering RNA; ERK, extracellular signal-regulated kinase; p-, phosphorylated.

A

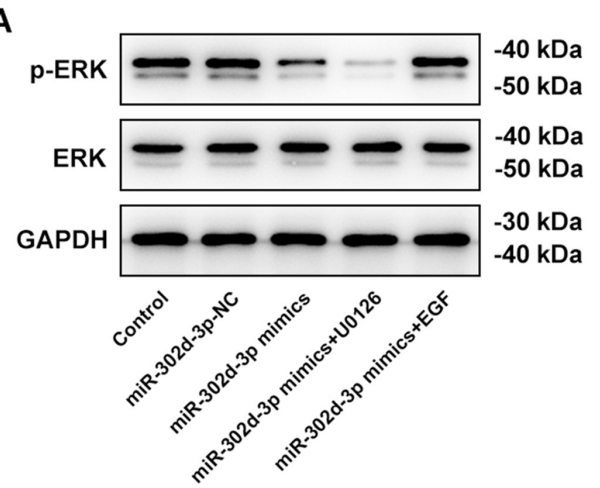

B

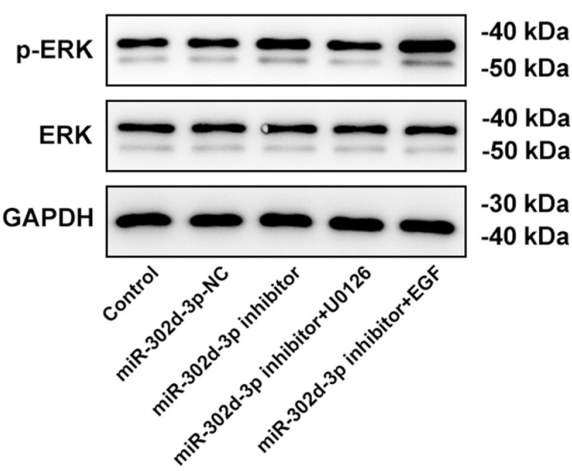

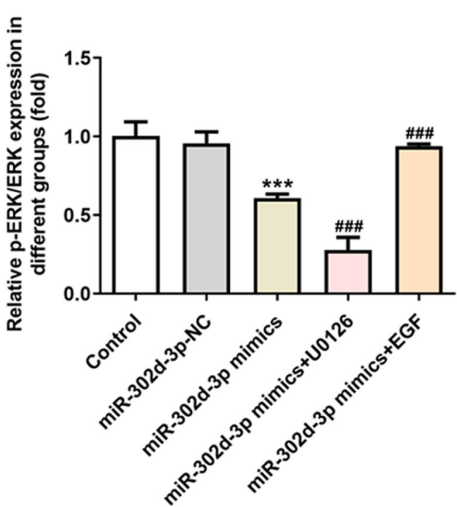

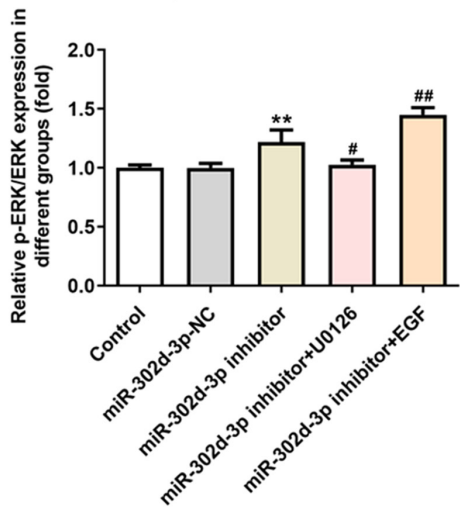

Figure 7. Expression of ERK pathway-related proteins following the addition of agonists and inhibitors. Western blotting was performed to detect the expression of ERK pathway-related proteins following transfection with (A) miR-302d-3p mimics or (B) miR-302d-3p inhibitor plus U0126 or EGF. ${ }^{* *} \mathrm{P}<0.01$, ${ }^{* * * *} \mathrm{P}<0.001$ vs. miR-302d-3p-NC; ${ }^{*} \mathrm{P}<0.05,{ }^{\# \#} \mathrm{P}<0.01$, ${ }^{\# \#} \mathrm{P}<0.001$ vs. miR-302d-3p mimics or inhibitor. miR, microRNA; NC, negative control; ERK, extracellular signal-regulated kinase; $\mathrm{p}-$, phosphorylated. 
A

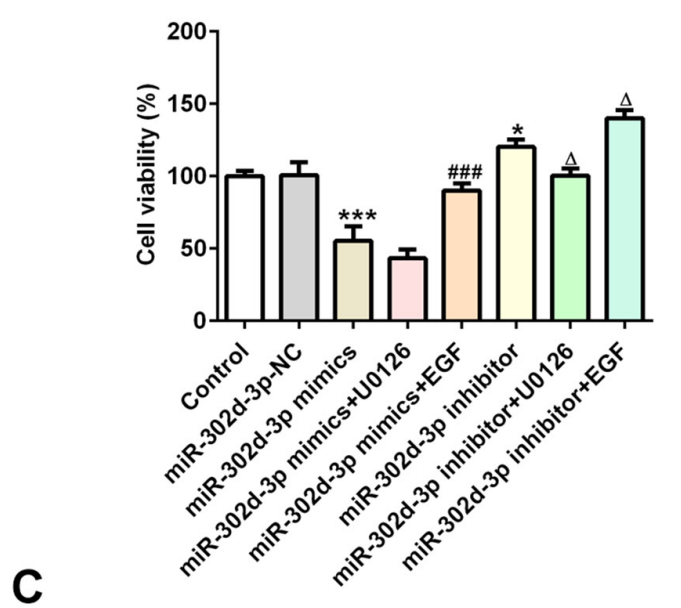

B

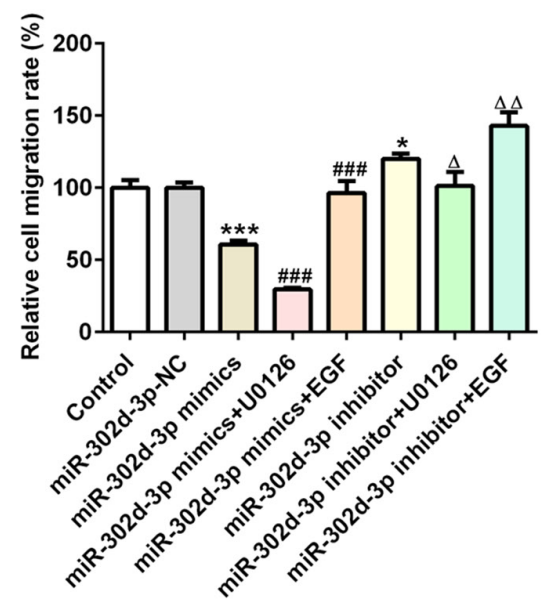

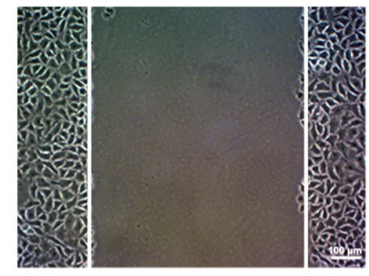
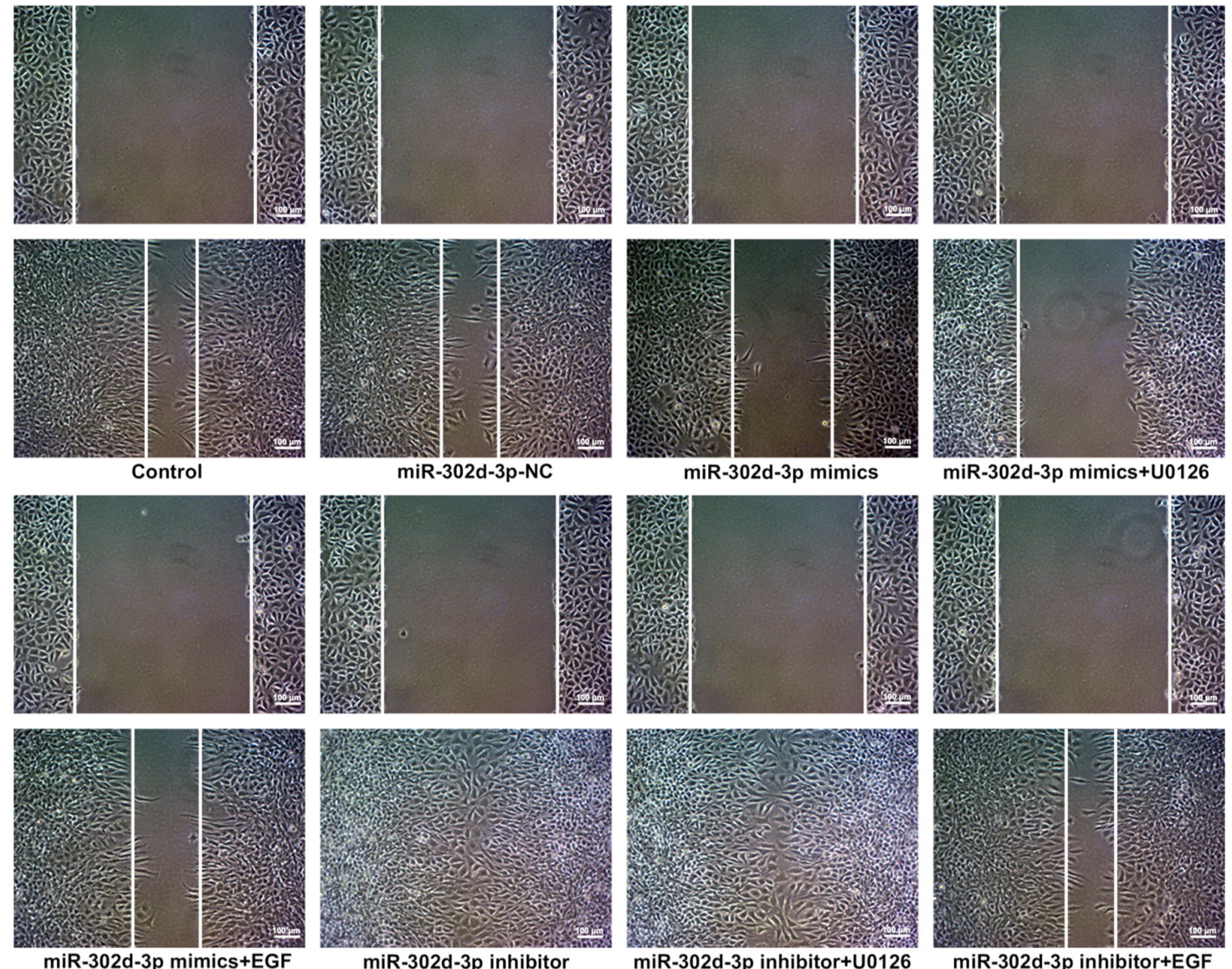

miR-302d-3p inhibitor+U0126
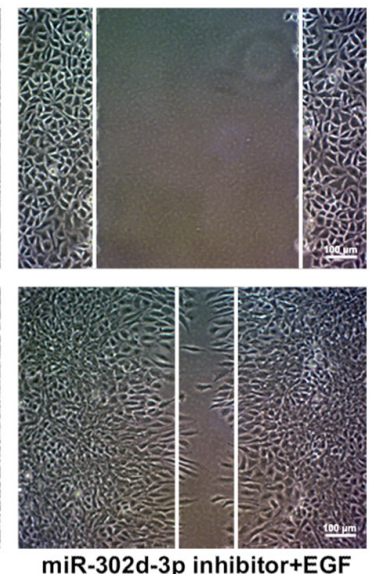

Figure 8. miR-302d-3p regulates ERK signaling pathway by targeting transmembrane Bax inhibitor motif containing 6 to inhibit the viability and migration of breast cancer cells. (A) Cell Counting Kit-8 assay was performed to determine cell viability. (B and C) A wound healing assay was conducted to measure cell migration (scale bar, $100 \mu \mathrm{m}$ ). ${ }^{*} \mathrm{P}<0.05,{ }^{* * * *} \mathrm{P}<0.001$ vs. miR-302d-3p-NC; ${ }^{\# \# \#} \mathrm{P}<0.001$ vs. miR-302d-3p mimics; ${ }^{\Delta} \mathrm{P}<0.05,{ }^{\Delta \Delta} \mathrm{P}<0.01 \mathrm{vs}$. miR-302d-3p inhibitor. miR, microRNA; NC, negative control; ERK, extracellular signal-regulated kinase.

ERK protein may be a regulatory target for miR-302d-3p. Moreover, overexpression of TMBIM6 can activate the ERK signaling pathway in order to inhibit apoptosis (22). It was observed that, after promoting the expression of miR-302d-3p, the expression of p-ERK decreased, while further inhibiting the expression of TMBIM6 further decreased the expression of $\mathrm{p}$-ERK. When inhibiting the expression of miR-302d-3p, p-ERK increased, while upon further inhibition of TMBIM6, the expression of $\mathrm{p}$-ERK decreased. Therefore, these results suggested that miR-302d-3p may regulate the ERK signaling pathway by targeting TMBIM6 to affect the viability, migration and apoptosis of $\mathrm{BC}$ cells. Addition of the inhibitor U0126 further aggravated the miR-302d-3p-induced decrease in cell viability and migration. Furthermore, U0126 could further increase miR-302d-3p-induced apoptosis, whereas this phenomenon was reversed following the addition of an EGF agonist. These results indicated that miR-302d-3p may regulate the ERK signaling pathway via targeting TMBIM6 to inhibit viability and migration and promote apoptosis of BC cells. 
A

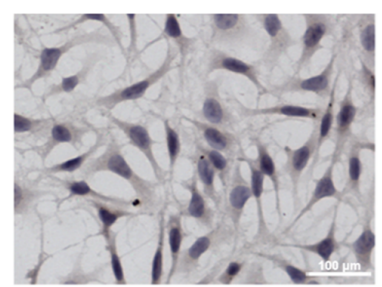

Control

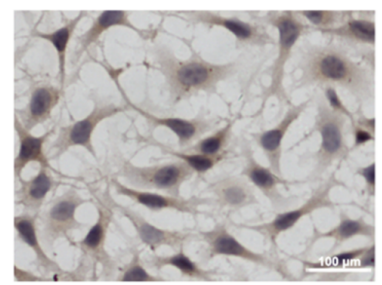

miR-302d-3p mimics+EGF

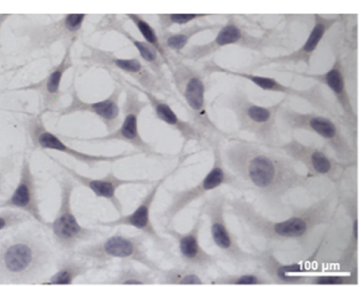

miR-302d-3p-NC

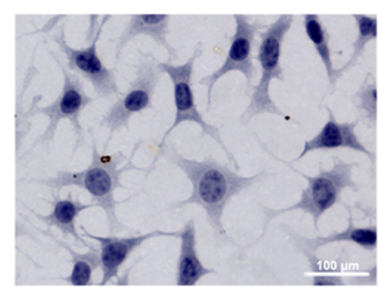

miR-302d-3p inhibitor

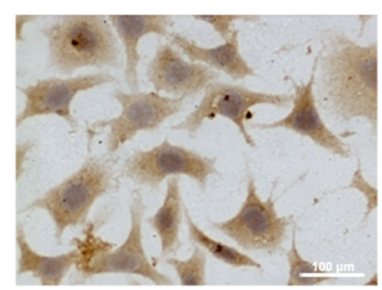

miR-302d-3p mimics

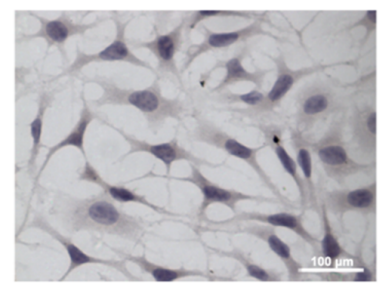

miR-302d-3p inhibitor+U0126 miR-302d-3p inhibitor+EGF

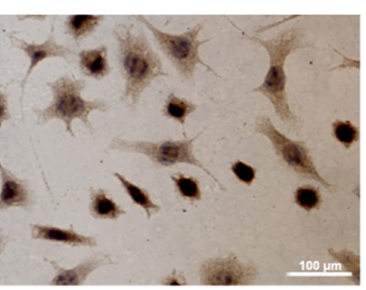

miR-302d-3p mimics+U0126

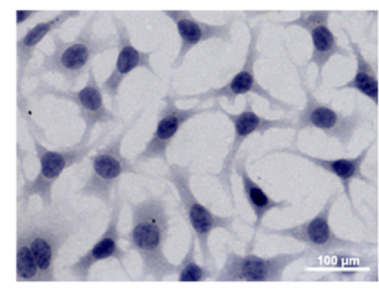

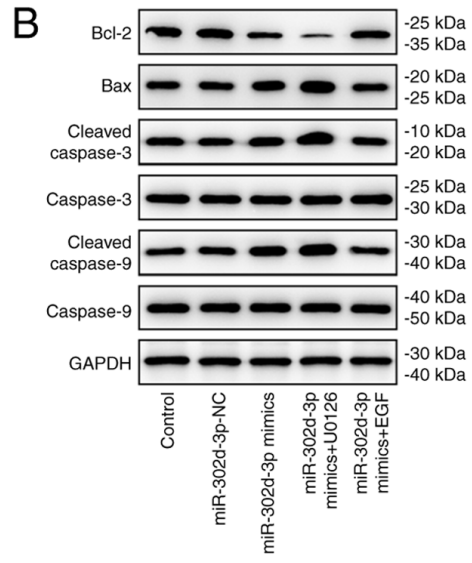
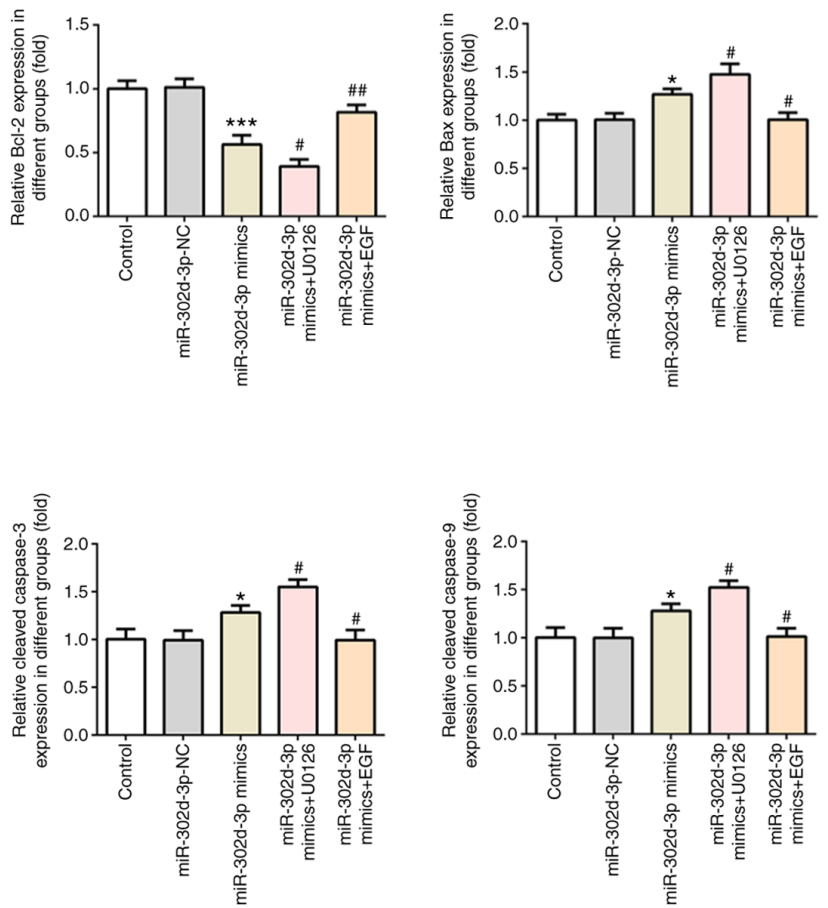

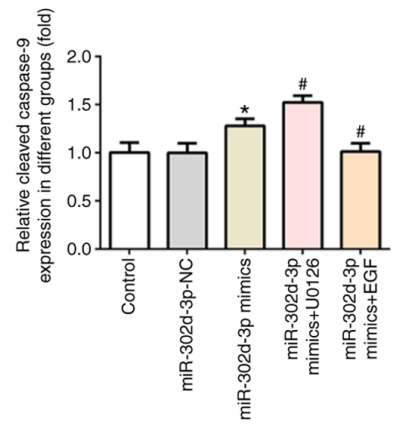

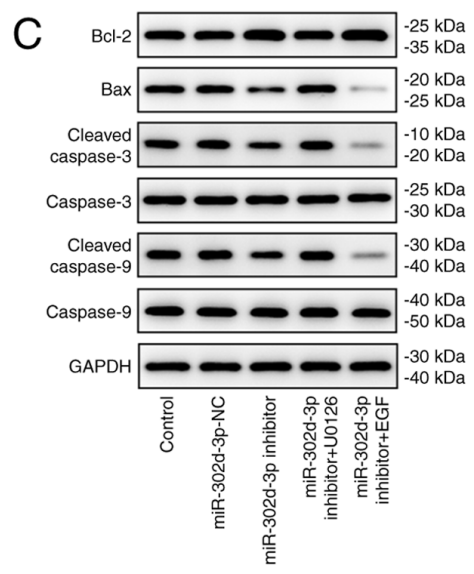
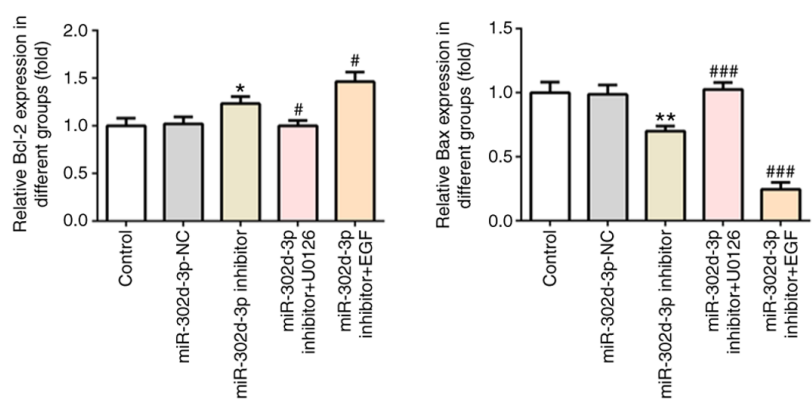

Figure 9. miR-302d-3p regulates ERK signaling pathway by targeting transmembrane Bax inhibitor motif containing 6 to promote apoptosis of BC cells. (A) TUNEL assay was performed to detect the apoptosis rate of BC cells (scale bar, $100 \mu \mathrm{m}$ ). Western blotting was performed to measure the expression of apoptosis-related proteins following transfection with (B) miR-302d-3p mimics or (C) miR-302d-3p inhibitor plus U0126 or EGF. ${ }^{*} \mathrm{P}<0.05$, ${ }^{* *} \mathrm{P}<0.01$, ${ }^{* * *} \mathrm{P}<0.001$ vs. miR-302d-3p-NC; ${ }^{\#} \mathrm{P}<0.05$, ${ }^{\# \#} \mathrm{P}<0.01,{ }^{\# \#} \mathrm{P}<0.001$ vs. miR-302d-3p mimics or inhibitor. miR, microRNA; NC, negative control; ERK, extracellular signal-regulated kinase; $\mathrm{BC}$, breast cancer. 
In conclusion, the present study revealed that miR-302d-3p regulated the viability, migration and apoptosis of $\mathrm{BC}$ cells via the regulation of TMBIM6-mediated ERK signaling, which may provide a novel experimental basis for targeted gene therapy for BC.

\section{Acknowledgements}

Not applicable.

\section{Funding}

No funding was received.

\section{Availability of data and materials}

The datasets used and/or analyzed during the current study are available from the corresponding author on reasonable request.

\section{Authors' contributions}

YL made substantial contributions to the conception and design of the study, and the acquisition of data. ZQ and LB made substantial contributions to analysis and interpretation of data. YL and ZQ confirm the authenticity of all the raw data. All authors read and approved the final manuscript.

\section{Ethics approval and consent to participate}

The study protocol was approved by the Baoan Central Hospital of Shenzhen (Shenzhen, China), and all patients provided written informed consent. All of the procedures were in compliance with The Declaration of Helsinki and relevant policies in China.

\section{Patient consent for publication}

Not applicable.

\section{Competing interests}

The authors declare that they have no competing interests.

\section{References}

1. Anastasiadi Z, Lianos GD, Ignatiadou E, Harissis HV and Mitsis M: Breast cancer in young women: An overview. Updates Surg 69: 313-317, 2017.

2. Merino Bonilla JA, Torres Tabanera M and Ros Mendoza LH: Breast cancer in the 21st century: From early detection to new therapies. Radiologia 59: 368-379, 2017.

3. Tian Z, Tang J, Liao X, Yang Q, Wu Y and Wu G: An immune-related prognostic signature for predicting breast cancer recurrence. Cancer Med 9: 7672-7685, 2020.

4. Li Y, Huo J, Pan X, Wang C and Ma X: MicroRNA $302 b-3 p / 302 c-3 p / 302 d-3 p$ inhibits epithelial-mesenchymal transition and promotes apoptosis in human endometrial carcinoma cells. Onco Targets Ther 11: 1275-1284, 2018.

5. Erratum: MicroRNA 302b-3p/302c-3p/302d-3p inhibits epithelial-mesenchymal transition and promotes apoptosis in human endometrial carcinoma cells [Erratum]. Onco Targets Ther 11: 2203,2018
6. Ecevit CO, Aktas S, Tosun Yildirim H, Demira $\breve{g}$ B Erbay A, Karaca İ, Çelik A, Demir AB, Erçetin AP and Olgun N: MicroRNA-17, MicroRNA-19b, MicroRNA-146a, MicroRNA-302d expressions in hepatoblastoma and clinical importance. J Pediatr Hematol Oncol 41: 7-12, 2019.

7. Chen D and Yang H: Integrated analysis of differentially expressed genes in breast cancer pathogenesis. Oncol Lett 9: 2560-2566, 2015

8. Livak KJ and Schmittgen TD: Analysis of relative gene expression data using real-time quantitative PCR and the 2(-Delta Delta C(T)) method. Methods 25: 402-408, 2001.

9. Wang S, Zheng Y, Hu Z, Wang Z, Zhang Y and Wei L: Downregulated miR302d3p promotes chondrocyte proliferation and migration by regulation of Unc-51-like kinase 1 . Int $\mathrm{J}$ Mol Med 44: 1039-1047, 2019.

10. Xu J, Wang Y, Hua X, Xu J, Tian Z, Jin H, Li J, Wu XR and Huang C: Inhibition of PHLPP2/cyclin D1 protein translation contributes to the tumor suppressive effect of NFкB2 (p100). Oncotarget 7: 34112-34130, 2016

11. Wang F, Yang L, Sun J, Zheng J, Shi L, Zhang G and Cui N: Tumor suppressors microRNA-302d and microRNA-16 inhibit human glioblastoma multiforme by targeting NF- $\mathrm{kB}$ and FGF2. Mol Biosyst 13: 1345-1354, 2017.

12. Ishikawa T, Watanabe N, Nagano M, Kawai-Yamada $M$ and Lam E: Bax inhibitor-1: A highly conserved endoplasmic reticulum-resident cell death suppressor. Cell Death Differ 18: 1271-1278, 2011.

13. Xu Q and Reed JC: Bax inhibitor-1, a mammalian apoptosis suppressor identified by functional screening in yeast. Mol Cell 1: 337-346, 1998.

14. Junjappa RP, Kim HK, Park SY, Bhattarai KR, Kim KW, Soh JW, Kim HR and Chae HJ: Expression of TMBIM6 in cancers: The involvement of Sp1 and PKC. Cancers (Basel) 11: 974, 2019.

15. Lee GH, Chae HJ and Kim HR: Monoamine carboxylate transporters are involved in BI-1-associated cancer metastasis in HT1080 colon fibrosarcoma cells. Int J Oncol 39: 209-216, 2011.

16. Li D, Wei Y, Xu S, Niu Q, Zhang M, Li S and Jing M: A systematic review and meta-analysis of bidirectional effect of arsenic on ERK signaling pathway. Mol Med Rep 17: 4422-4432, 2018.

17. Yang C, Yu H, Chen R, Tao K, Jian L, Peng M, Li X, Liu M and Liu S: CXCL1 stimulates migration and invasion in ERnegative breast cancer cells via activation of the ERK/MMP2/9 signaling axis. Int J Oncol 55: 684-696, 2019.

18. Sheng W, Chen C, Dong M, Wang G, Zhou J, Song H, Li Y, Zhang $\mathrm{J}$ and Ding S: Calreticulin promotes EGF-induced EMT in pancreatic cancer cells via Integrin/EGFR-ERK/MAPK signaling pathway. Cell Death Dis 8: e3147, 2017.

19. Maehara O, Suda G, Natsuizaka M, Ohnishi S, Komatsu Y, Sato F, Nakai M, Sho T, Morikawa K, Ogawa K, et al: Fibroblast growth factor-2-mediated FGFR/Erk signaling supports maintenance of cancer stem-like cells in esophageal squamous cell carcinoma. Carcinogenesis 38: 1073-1083, 2017.

20. Buffet C, Hecale-Perlemoine K, Bricaire L, Dumont F, Baudry C, Tissier F, Bertherat J, Cochand-Priollet B, Raffin-Sanson ML, Cormier F and Groussin L: DUSP5 and DUSP6, two ERK specific phosphatases, are markers of a higher MAPK signaling activation in BRAF mutated thyroid cancers. PLoS One 12: e0184861, 2017.

21. Zhao L, Wang Y,Jiang L, He M, Bai X, Yu L and Wei M: MiR-302a/ $\mathrm{b} / \mathrm{c} / \mathrm{d}$ cooperatively sensitizes breast cancer cells to adriamycin via suppressing P-glycoprotein(P-gp) by targeting MAP/ERK kinase kinase 1 (MEKK1). J Exp Clin Cancer Res 35: 25, 2016.

22. Kim JH, Lee ER, Jeon K, Choi HY, Lim H, Kim SJ, Chae HJ, Park SH, Kim S, Seo YR, et al: Role of BI-1 (TEGT)-mediated ERK1/2 activation in mitochondria-mediated apoptosis and splenomegaly in BI-1 transgenic mice. Biochim Biophys Acta 1823: 876-888, 2012.

This work is licensed under a Creative Commons Attribution-NonCommercial-NoDerivatives 4.0 International (CC BY-NC-ND 4.0) License. 